\title{
Oral desmopressin in central diabetes insipidus
}

\author{
U WESTGREN, C WITTSTRÖM, AND A S HARRIS
}

Department of Pediatrics, University Hospital, Lund, and Faculty of Pharmacy, Biomedicum, Uppsala, Sweden

SUMMARY Seven paediatric patients with central diabetes insipidus were studied in an open dose ranging study in hospital followed by a six month study on an outpatient basis to assess the efficacy and safety of peroral administration of DDAVP (desmopressin) tablets. In the dose ranging study a dose dependent antidiuretic response was observed. The response to $12 \cdot 5-50 \mathrm{mcg}$ was, however, less effective in correcting baseline polyuria than were doses of $100 \mathrm{mcg}$ and above. Patients were discharged from hospital on a preliminary dosage regimen ranging from 100 to $400 \mathrm{mcg}$ three times daily. After an initial adjustment in dosage in three patients at one week follow up, all patients were stabilised on treatment with tablets and reported an adequate water turnover at six months. As with the intranasal route of administration dosage requirements varied from patient to patient, and a dose range rather than standard doses were required. A significant correlation, however, was found for the relation between previous intranasal and present oral daily dosage.

No adverse reactions were reported. No clinically significant changes were noted in blood chemistry and urinalysis. All patients expressed a preference for the oral over existing intranasal treatment. Treatment with tablets offers a beneficial alternative to the intranasal route, particularly in patients with chronic rhinitis or impaired vision.

Diabetes insipidus in children is a rare disease caused by a deficiency of the antidiuretic hormone, vasopressin. This deficiency leads to a failure to conserve water and results in a polyuria and compensatory polydipsia. Due to the inability to concentrate urine, the child with untreated diabetes insipidus will have high urinary losses, and if treatment is not begun promptly there is a danger of rapid dehydration. If the disease is untreated or badly controlled the child can develop chronic dehydration, unexplained fever, vomiting, constipaion, and failure to thrive. These may lead to serious neurological disturbances not always corrected when substitution treatment is started.

The aetiology of diabetes insipidus is most often a destructive process along the hypothalamopituitary axis. In childhood the cause of this damage is often a tumour - that is, craniopharyngioma. Consequently, diabetes insipidus will often develop in a child operated on for a pituitary tumour or one located near the pituitary. ${ }^{1}$ In addition, many of these children have other pituitary dysfunctions and also impairment of vision due to lesions of the optic tracts.
Diabetes insipidus was earlier treated with vasopressin and its analogues such as lysine vasopressin or mixtures of lysine and arginine vasopressin. These drugs, however, produce several side effects, partly due to their pressor activity. ${ }^{2}$

DDAVP (1-desamino-8-d-arginine vasopressin acetate; Ferring, UK), a synthetic analogue of vasopressin, was introduced more than 10 years ago. Since then, experimental and clinical experience has shown that desmopressin is the drug of choice for the treatment of central diabetes insipidus. Compared with the drugs used earlier, it is characterised by a potent antidiuretic action, minimal side effects, and absence of pressor activity. ${ }^{3-6}$

At present desmopressin is available for intravenous and intranasal administration. The nasal form is delivered by a plastic nasal tube and involves a technique that is difficult to teach small children correctly. As many of the patients with diabetes insipidus are partially sighted they are sometimes unable to use the tube themselves and have to rely on their parents. Moreover, the nasal mode of delivery can be compromised by nasal congestion seen during allergic rhinitis or common 
colds. Another disadvantage with the nasal form of administration is that the aqueous desmopressin solution must be kept in a refrigerator, which presents practical problems for patients at school and when travelling away from home.

For these reasons other methods of delivering desmopressin have been tested, such as a nasal pump and sublingual lozenges. ${ }^{7}$ Although sublingual administration does work, this form of administration is unsuitable in paediatric practice.

The possibility of delivering desmopressin perorally as a tablet has received little attention due to the common opinion that peptides are completely degradated by enzymes in the gastrointestinal tract. As early as 1913, however, there was a report of a successful treatment of a patient with diabetes insipidus by eating two to seven fresh pituitary bodies daily from cattle. ${ }^{8}$ It is also known that diand tripeptides may be absorbed uncleaved in the intestine of man. ${ }^{9} 10$

Recent studies have shown that desmopressin administered as an aqueous solution perorally caused an antidiuretic response in dogs and humans. ${ }^{11} 12$ These facts led to a study in adult patients where it was described that a daily oral dosage of $100-200 \mathrm{mcg}$ three times daily was sufficient to control polyuria. ${ }^{13}$ Against this background, we designed a study to evaluate the efficacy and safety of oral desmopressin in paediatric patients and to obtain information on the correlation between intranasal and oral dosing.

\section{Materials and methods}

Patients. Seven patients (six boys and one girl) with central diabetes insipidus were studied. Ages ranged from 4 to 18 years (mean 12.6 years). All had diabetes insipidus secondary to tumours, and six had been operated on for a craniopharyngioma and one for a pituitary adenoma. The diagnosis of diabetes insipidus had previously been confirmed by a standard water deprivation test. Patients were being treated with standard daily doses of intranasal desmopressin ranging from 5-60 mcg (mean 25 $\mathrm{mcg}$ ). In addition, all patients were receiving concomitant treatment with drugs for other pituitary deficiencies, including thyroxine, cortisone acetate, and growth hormone. Three patients had impaired vision secondary to the tumour or the treatment of the tumour. After thorough information about the study had been given, written informed consent was obtained from parents. The study protocol was approved by the hospital's ethics committee.

Pretreatment period. One week before admission to hospital the patients were asked to record dose, diuresis, and body weight while on treatment with intranasal desmopressin.

Dose ranging study. Each child was admitted to hospital for a maximum of five nights. On admission the child was given the usual intranasal dose of desmopressin. The antidiuretic effect was then followed until the pharmacological effect had disappeared and the basal polyuria and low urine osmolality were established.

Patients were then given desmopressin tablets in single rising doses of $12 \cdot 5,25,50,100,200$, and 400 mcg desmopressin. (Minirin, Ferring Pharmaceuticals, Malmö, Sweden).

The patient's response to each single rising dose was monitored by following urine output and osmolality each 30 minutes for the first two hours and at hourly intervals thereafter. The next assigned dose was given when at least $60 \%$ of basal polyuria was reached. Serum osmolality, sodium concentration, blood pressure, and heart rate were determined immediately before and 30 minutes after each new dose. At the end of the hospital period, an analysis was made to determine the optimum dose at which a therapeutic response was obtained to ensure an antidiuretic effect for at least eight hours.

Outpatient follow up. After discharge from hospital the patients continued treatment based on a three times daily dosage regimen. Each patient was provided with a diary in which dose, diuresis, and body weight were recorded at least two days a week for the first four weeks and each third month thereafter.

Any adjustments to the dosage regimen were made either after telephone contact or at regular visits at weeks $1,2,3,4,8,12$, and 24 . In addition to body weight and vital signs, the following laboratory tests were performed at baseline while on intranasal treatment and at four, 12, and 24 weeks on tablets: blood chemistry, urinalysis, serum electrolytes concentrations, and liver function.

Statistics. Linear regression was performed by the method of least squares. All laboratory data were analysed using Student's $t$ test for paired observations.

\section{Results}

Dose ranging study. Before the first oral dose, the range of diuresis when in polyuria was $4-14 \mathrm{l} / 24 \mathrm{~h}$ (mean 8.8).

Figure 1 shows the effect of each dose on urine osmolality. Doses of $50 \mathrm{mcg}$ and below were less effective in correcting baseline urine osmolality. For doses of $100 \mathrm{mcg}$ and above, however, an increase in 


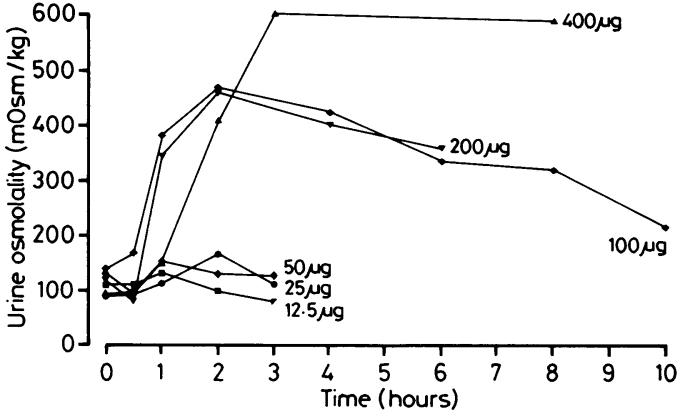

Fig. 1 The effect of single rising doses of desmopressin tablets on urine osmolality in seven patients (mean).

duration and magnitude of response is seen for each rising dose. For each patient an individual dose response analysis was performed by calculating the maximum urine osmolality after each dose (Figure 2). A significant correlation was observed between $\log$ maximum urine osmolality and log desmopressin dose by body weight $(r=0 \cdot 61, p<0 \cdot 05)$.

The response to $100 \mathrm{mcg}$ doses and above gave rise to a more concentrated urine osmolality, and most patients could be discharged from hospital with an initial dosage regimen of 100-200 mcg desmopressin three times daily. An exception was case 3, a 15 year old male, body weight $95 \mathrm{~kg}$, who was started on $400 \mathrm{mcg}$ desmopressin three times daily.

No adverse reactions to the administration of desmopressin at any dose level were observed. No clinically significant changes were noted in body weight, blood pressure, or heart rate.

Outpatient follow up. During the first few weeks of treatment small dosage adjustments were made for

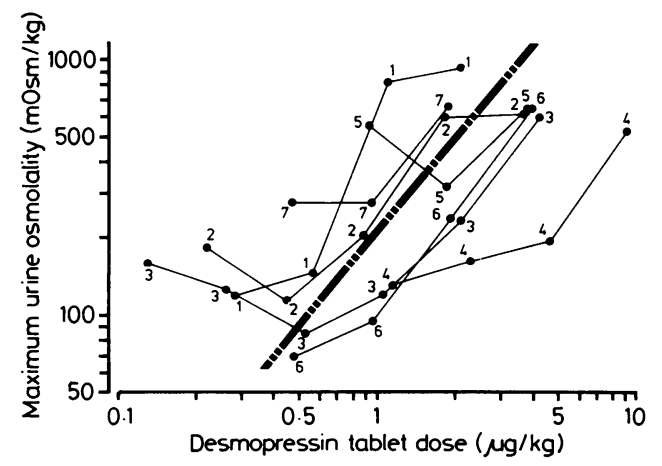

Fig. 2 The maximum response in urine osmolality to single rising doses of desmopressin tablets in seven cases $(1-7)$. Coefficient of correlation $r=0.61 \quad(p<0.05)$.

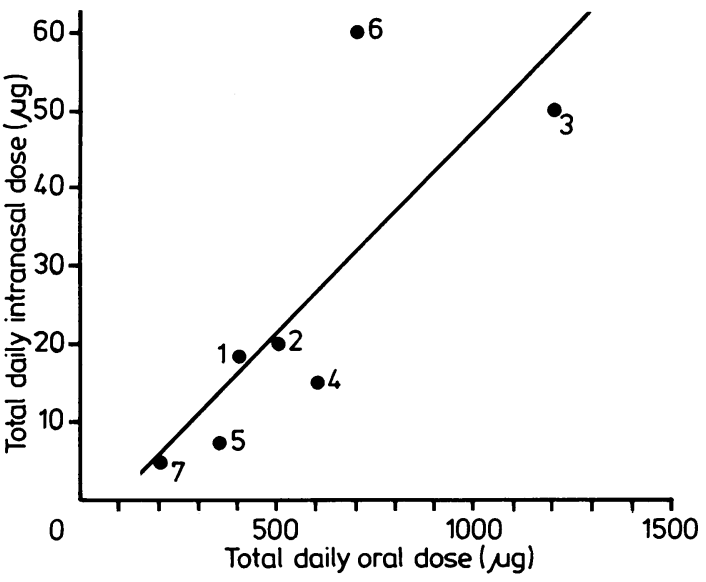

Fig. 3 Correlation between daily intranasal and oral doses in seven cases (1-7). Coefficient of correlation $r=0.79$ $(p<0 \cdot 01)$.

three patients, after which all were stabilised on treatment at six months follow up. During this period daily diuresis was similar to diuresis during the period on intranasal treatment. The mean daily dosage was $550 \mathrm{mcg}$ (range: $100 \mathrm{mcg}$ twice daily to $400 \mathrm{mcg}$ three times daily). In comparison, the previous mean intranasal dosage had been $25 \mathrm{mcg}$ (range: $5 \mathrm{mcg}$ nightly to $20 \mathrm{mcg}$ three times daily). Figure 3 shows a linear correlation between previous daily intranasal doses and current daily tablet doses $(\mathrm{r}=0.79, \mathrm{p}<0.05)$. At six month follow up no clinically significant changes in laboratory safety variables were recorded and no adverse reactions were observed.

\section{Discussion}

Since its introduction desmopressin has been the drug of choice in the treatment of diabetes insipidus, although the mode of administration, intranasally with a tube, has several practical disadvantages. Most children treated with desmopressin have a central diabetes insipidus secondary to a brain tumour located in the hypothalamopituitary region. ${ }^{1}$ In addition, they are often severely handicapped by impaired vision, which leads to difficulties in handling the nasal tube. Consequently, there are practical advantages to be gained by switching to oral treatment.

The present study confirms an earlier investigation in adult patients showing that peroral administration of desmopressin results in an antidiuretic response that is valuable in clinical practice. ${ }^{13}$ Although the individual response varied greatly 
between patients, a linear correlation was observed between dose and urine osmolality. A similar correlation was reported by Moses et al after desmopressin had been administered subcutaneously in doses of $0.5-4.0 \mathrm{mcg}$ whereby doubling the dose lengthened its action by four hours. ${ }^{14}$

In this study, a mean antidiuretic activity of eight hours was achieved at doses of $100-400 \mathrm{mcg}$. The patients showed a great interindividual variation both in magnitude and duration of response, but the dose range was found to be of the same order as for intranasal desmopressin, which had previously been administered in daily doses of 5-60 mcg. The aetiology of this wide range is probably due to the variation in secretion of endogenous vasopressin.

As with the intranasal route of administration individual dosage requirements will vary, and a standard dose schedule is not possible. A significant correlation was found, however, between the intranasal and the oral dose indicating a ratio of 1:20. A suitable oral dose for most patients will probably be in the range of $100-400 \mathrm{mcg}$ three times daily. Some of our patients switched from twice daily intranasal administration to three times daily oral administration. In our experience, however, the children did not find this to be a serious drawback.

Compared with the peroral dose used by Vilhardt et al,${ }^{13}$ our daily dose is higher. This could possibly be explained by different kinetics between adults and children or by differences in patient selection. It is most likely, however, to be due to more practical aspects, such as the higher water turnover in adults, whereby higher fluid losses are compensated by extra water intake (Hammer, personal communication). Moreover, as children need more sleep at night than adults a larger dose at bedtime was given to minimise night time diuresis and ensure adequate duration of sleep.

It should be emphasised that it is important to follow the patient's urinary output closely, especially during changes in treatment. One reason for this is that the children and parents in our experience have a tendency to underestimate the child's urinary output, which implies that many patients may be undertreated.

This study shows that treatment with desmopressin tablets is effective and safe for diabetes insipidus in children. All children expressed a preference for the tablets. The doses needed to control polyuria are higher than with intranasal administration, but this is outweighed by the numerous advantages of the tablet form. Our patients have now been on continuous treatment with tablets for 10 months. To date, we have not observed any loss of efficacy or side effects.

We thank Astrid Hansson and Andrew Szelke for their technical help. Samples of desmopressin tablets (Minirin) were kindly provided by Ferring Pharmaceuticals, Malmö. Sweden. At present the tablets are not commercially available.

\section{References}

1 Czernichow P. Pomarede R, Brauner R, Rappaport R. Neurogenic diabetes insipidus in children. Horm Res 1985;13: 190-209.

2 Andersson K-E, Arner B, Furst E, Hedner P. Antidiuretic response to hypertonic saline infusion, water deprivation, and a synthetic analogue of vasopressin in patients with hereditary, hypothalamic diabetes insipidus. Acta Med Scand 1974;195: 17-23.

${ }^{3}$ Andersson K-E, Arner B. Effects of DDAVP, a synthetic analogue of vasopressin in patients with cranial diabetes insipidus. Acta Med Scand 1972:192:21-7.

+ Seif SM, Zenser TV, Ciarochi FF, Davis BB, Robinson AG. DDAVP (1-Desamino-8-D-Arginine-vasopressin) treatment of central diabetes insipidus-mechanism of prolonged antidiuresis. $J$ Clin Endocrinol Metab 1978;46:381-8.

5 Robinson AG. DDAVP in the treatment of central diabetes insipidus. N Engl J Med 1976;294:507-11.

" Cobb WE, Spare A, Reichlin S. Neurogenic diabetes insipidus: management with DDAVP (1-Desamino-8-D-ArginineVasopressin). Ann Intern Med 1978;88:183-8.

7 Grossman A. Fabbri A. Goldberg PL. Bessar GM. Two new modes of desmopressin (DDAVP) administration. $\mathrm{Br} \mathrm{Med} \mathrm{J}$ 1980;ii: 1215 .

${ }^{x}$ Velden $\mathrm{R}$ von den. Die nierenwirkung von hypophysenextrak ten beim menschen. Berl klin Wschr 1913;2:2083.

"Adibi SA. Morse EL. Intestinal transport of dipeptides in man: relative importance of hydrolysis and intact absorption. J Clin Invest 1971;50:2266-75.

11) Saffran M. Franco-Saenz R, Kong A. Papahadjopoulos D, Szoka F. A model for the study of the oral administration of peptide hormones. Can J Biochem 1979:57:548-53.

1 Vilhardt H. Bic P. Antidiuretic response in conscious dogs following peroral administration of arginine vasopressin and its analogues. Eur $J$ Pharmacol 1983;93:201-4.

12 Vilhardt H, Hammer M, Bic P. Peroral administration of antidiuretic peptides to conscious dogs, normal humans and patients with diabetes insipidus. Horm Res 1985:13:314-20.

13 Hammer M, Vilhardt H. Peroral treatment of diabetes insipidus with a polypeptide hormone analog, desmopressin. J Pharmacol Exp Ther 1985;234:754-60.

14 Moses AM. Moses LK, Nostman DD. Springer J. Antidiuretic responses to injected desmopressin, alone and with indomethacin. $J$ Clin Endocrinol Metab 1981:52:910-3.

Correspondence to Dr U Westgren, Department of Pediatrics, University Hospital. S-221 85 Lund, Sweden.

Received 18 November 1985 